\title{
Pour une analyse dispositionnelle des pratiques professionnelles
}

\author{
David Pichonnaz ${ }^{1}$, Kevin Toffel $^{2}$
}

Si l'étude du travail est ancienne, l'analyse sociologique des groupes professionnels est en expansion en France et d'une manière générale dans le monde francophone depuis les années 1980 (Demazière, Gadea, 2009 ; Dubar, Tripier, Boussard, 2011)³. Comme toute entreprise de spécialisation - conséquence d'une parcellisation de la discipline en sociologies thématiques - ce mouvement court le risque d'un phénomène de repli de la part des chercheurs sur les objets « travail » et " professions ». Cette focalisation porte en elle au moins deux inconvénients. D'une part, elle a tendance à laisser de côté des enjeux de portée générale pour la sociologie, d'autant que les travaux en question se concentrent souvent sur une profession en particulier. D'autre part, elle conduit à ignorer les liens unissant les univers professionnels aux autres sphères sociales. Or, on ne peut bien saisir la position qu'occupent les individus au sein d'une profession, ni leurs prises de position tendanciellement correspondantes, en faisant l'économie de l'examen du social incorporé qu'ils y importent.

Partant de ce double constat, ce numéro consacré à l'analyse dispositionnelle des pratiques professionnelles souhaite se réconcilier avec une sociologie générale tout en se concentrant sur la question particulière des origines et des trajectoires sociales conduisant non seulement aux professions, mais aussi à des positions (horizontales ou verticales) occupées au sein de celles-ci. On y trouvera des articles s'intéressant à la manière dont des dispositions acquises hors de la sphère du travail, en particulier au cours de la socialisation primaire, contribuent à façonner les carrières, les visions du métier et les pratiques professionnelles. L'analyse dispositionnelle des pratiques professionnelles, indissociable d'une approche structurale du travail, nous semble à même de rendre compte de l'ancrage social des professionnels. C'est ce que montrent les articles réunis ici et ce dont nous rendons compte dans la première partie de cette introduction. Dans une seconde partie, nous interrogeons la manière dont les espaces professionnels (re)façonnent les individus, en articulant l'étude de la socialisation primaire et celle de la socialisation professionnelle.

\footnotetext{
${ }^{1}$ Haute école de travail social (HES-SO Valais-Wallis).

${ }^{2}$ Haute école de santé Vaud (HES-SO Haute école spécialisée de Suisse occidentale).

${ }^{3}$ Nous remercions vivement Marc Perrenoud et Muriel Surdez pour leur relecture attentive d'une version antérieure de ce texte. II va de soi que les propos énoncés dans cet article n'engagent cependant que ses auteurs.
} 


\section{Pour une approche structurale du travail}

Attendu « l'inscription des structures sociales dans les corps » (Bourdieu, 1997 : 206), c'est bien de société faite corps dont on parle lorsqu'on adopte une approche dispositionnelle pour saisir les propensions qu'ont les individus «à penser, à sentir et à agir ", produits de leurs « expériences socialisatrices multiples» (Lahire, 2002 : 3). Aussi, il convient d'emblée de relever qu'opter pour une analyse dispositionnelle consiste à adopter une perspective déterministe, non mécaniste, du monde social, et à placer les pratiques et les représentations des agents au centre de l'analyse ; au détriment des processus de constitution des "identités professionnelles» (Dubar, 2009). On ne peut repérer des dispositions que dans les traces que laissent des stratégies d'un type bien particulier : celles qui peuvent « être objectivement conformes aux intérêts objectifs de leurs auteurs sans avoir été expressément conçue à cette fin » (Bourdieu, 1984 : 119-120). Ces tendances à l'action - ou sens pratique - sont donc au moins aussi largement inconscientes que conscientes comme le relève Romain Pudal dans la conclusion de ce numéro. C’est par conséquent une philosophie de l'action ancrée sur le principe de nonconscience qui, à l'encontre de l'agir relevant d'un choix rationnel et gouverné par des « motivations » énoncées et reconnues, « impose au contraire que l'on construise le système des relations objectives dans lesquelles les individus se trouvent insérés et qui s'expriment plus adéquatement dans l'économie ou la morphologie des groupes que dans les opinions et les intentions déclarées des sujets 》 (Bourdieu, Chamboredon, Passeron, 1968 : 41). Au fond, il ne s'agit ici pas d'autre chose que de se demander pourquoi les individus pensent ce qu'ils pensent, disent ce qu'ils disent et font ce qu'ils font et, peut-être surtout, pourquoi ils ne pensent, disent et ne font pas autre chose, quand bien même les finalités de leurs actions leur échappent la plupart du temps.

On le voit, les réflexions proposées dans ce numéro s'inscrivent dans le sillage d'un questionnement qui constitue l'un des fondements du projet sociologique : celui d'étudier les relations nouant individus et société. Les articles qui le composent relèvent tous d'une analyse structurale qui s'intéresse aux déterminismes sociaux et historiques pesant sur les individus et participant à façonner des trajectoires, elles-mêmes intimement liées à des manières de voir le monde et de s'y voir. Car ce n'est qu'en prenant en compte les effets d'un social incorporé que l'on se donne les moyens de saisir les manières dont les individus perçoivent et investissent les univers qu'ils traversent : ici, les univers professionnels.

\subsection{Penser les professions comme des espaces sociaux}

Depuis l'article programmatique de R. Bucher et A. Strauss (1961) et l'apport avéré de la notion de «segment " pour désigner les sous-groupes qui composent les professions, on sait que ces dernières sont hétérogènes et composées d'entités ayant potentiellement 
des intérêts divergents ou étant associées à un différentiel de prestige ${ }^{4}$ - ce qu’E. C. Hughes a appelé la « division morale du travail » (Hughes, 1996). Cependant, comme le note F. Champy (2009), l'approche interactionniste, très attentive, face aux approches fonctionnalistes d'alors, à relever tant la diversité que la conflictualité des segments, omet de regarder ce qui unit ces sous-groupes professionnels. En outre, la question des limites et contours d'une profession reste largement inexplorée par cette approche et, partant, par les études sur le travail, de même que les relations entre la sphère du travail et les autres sphères sociales. On peut pourtant penser, à la suite de F. Lebaron (2000) à propos des économistes, que la délimitation des frontières d'un groupe n'est pas aussi évidente que peut le laisser penser l'existence d'un nom associé à une profession, non moins que ses rapports aux espaces sociaux environnants.

Une manière de combler ces deux angles morts des approches classiques en sociologie des professions consiste à appréhender ces dernières comme des espaces professionnels en adoptant la théorie des champs de P. Bourdieu ${ }^{5}$. Dans cette optique, les professions doivent être considérées non pas comme des entités homogènes mais, au contraire, comme des espaces de concurrences entre des agents qui se différencient. Cette différenciation règle les positions occupées par les agents parmi l'ensemble des positions possibles, elles-mêmes déterminées par un état de cet espace social, notamment par les rapports de force qui le traversent et le structurent $t^{6}$. Les manières d'occuper ces positions dépendent pour leur part des trajectoires sociales des agents, c'est-à-dire de leurs dispositions acquises au travers de processus cumulatifs de socialisations. Adopter cette perspective théorique - c'est à dire avoir recours tant à un « cadre conceptuel » qu'à une " méthode d'analyse » (Pinell, 2012) - permet de penser à la fois l'unité et la diversité d'une profession, en montrant que les individus occupent des positions en lien avec la possession d'une quantité spécifique de ressources et qu'ils sont en lutte pour la définition des enjeux et frontières de cet espace particulier. Se saisir de cette approche comme d'un programme de recherche nécessite d'embrasser synchroniquement l'entièreté de l'« espace des points de vue » (Bourdieu, 1993) qui compose un espace

\footnotetext{
${ }^{4}$ Les études sont nombreuses. On pourra se référer à l'ouvrage de R. Bercot, S. Divay et C. Gadea (2012) pour accéder à un panorama de cette question dans différentes professions. ${ }^{5}$ Précisons qu'espace et champ ne sont pas des synonymes. Bien que basé sur plusieurs éléments clés de la théorie des champs - cf. par exemple Bourdieu (1991) - tout travail empirique qui s'inspire de cette boîte à outils conceptuelle pour une analyse des professions en termes d'espaces professionnels ne doit pas faire oublier les écarts qui peuvent s'observer entre l'objet étudié et les implications théoriques associées à l'application du concept de champ ; en particulier en ce qui concerne leur autonomie relative. C'est en effet une condition fondamentale de l'existence d'un champ que celle de la rupture vis-à-vis des enjeux des champs environnants afin de se constituer en « monde à part »(Bourdieu, 1998 : 103), élément qui fait parfois défaut aux professions. À ce sujet, voir par exemple les travaux de L. Mathieu (2000; 2007), A. Vauchez (2008), P. Pinell (2012) et K. Toffel (2018).

${ }^{6}$ Les représentations et pratiques professionnelles dépendent de la position dans la hiérarchie (légale ou symbolique) interne à une profession, de l'appartenance à un " segment » particulier, du lieu de travail et des types de « clients » qui vont avec, etc.
} 
professionnel. Cette perspective laisse alors apparaître des classes et fractions de classes au sein de ces groupes pour partie unis (autour d'un univers de pratiques particulières et d'éventuelles formes spécifiques de capitaux) et pour partie divisés (y compris à propos de ce qui compte dans cet univers).

Pour saisir une profession comme un espace, c'est-à-dire comme une entité dynamique et relationnelle, il faut s'attacher à comprendre son développement historique au travers des luttes qui sans cesse le font et le défont. P. Pinell montre ainsi que la structuration du champ médical s'est effectuée au travers de concurrences entre des sphères spécialisées de l'activité médicale et par les liens que ces sous-univers ont historiquement entretenus avec d'autres sphères d'activités - économiques, politiques, universitaires, etc. (Pinell, 2009). Cette démarche analytique permet en outre de saisir les effets différenciés d'un métier sur les représentations et pratiques des agents selon les positions occupées dans cet espace. L. Mathieu (2000) a ainsi mis en lumière les luttes de placement réglées selon des principes de hiérarchisations spécifiques à l'espace de la prostitution. Ces travaux montrent par ailleurs que saisir la structuration d'une profession nécessite de la réinscrire dans les rapports qu'elle entretient avec les champs et espaces environnants, et pas uniquement avec les autres groupes professionnels (Abbott, 1988). Ou encore, comme l'évoque M. Quijoux à propos de l'approche bourdieusienne, à considérer le travail « comme un espace supplémentaire de l'expression des divisions sociales qui structurent les sociétés contemporaines " (Quijoux, 2015: 55). Penser les professions comme des espaces implique en effet de les situer dans l'espace social global, et permet de comprendre les logiques agissantes issues d'autres univers de production symbolique. La réinscription de la profession infirmière au sein du champ médical permet par exemple de voir que les logiques de ce dernier déterminent largement les représentations et pratiques professionnelles des infirmières, et ce malgré des effets de socialisation et des enjeux propres à l'espace infirmier (Longchamp et al., 2018 ; Toffel, 2018). De même, les pratiques de cirque (cf. le texte d'Émilie Salaméro et Marie Cordier dans ce numéro) sont dépendantes de la structuration du champ artistique, et le capital culturel que les « Quants » - ces ingénieurs-mathématiciens actifs dans la banque - ont accumulé dans leurs familles et dans le champ académique est des plus rentables dans l'espace professionnel de la finance (cf. le texte de Marlène Bouvet).

On peut se demander pourquoi cette approche topologique des professions est encore rarement adoptée pour rendre compte des prises de position au sein d'espaces professionnels différenciés. Que l'on pense au récent manuel de N. Vezinat (2016) consacré à la sociologie des groupes professionnels. Elle y introduit bel et bien la notion d'espace professionnel pour penser le rapport qu'entretient cet espace aux institutions extérieures ou aux « clients », et ce dans la continuité des approches classiques en sociologie des professions. Mais cet usage étréci de la notion ne permet pas de saisir la différenciation interne aux professions, cristallisant des oppositions structurées tantôt autour 
d'enjeux spécifiques, tantôt en lien avec des enjeux exogènes lorsque l'espace professionnel en question est fortement hétéronome.

Un rapide examen socio-historique des développements de l'objet "profession » aux États-Unis comme en France permet d'amener quelques éléments de réponse au moindre intérêt qu'a eu P. Bourdieu et les chercheurs qui s'inscrivent dans son sillage pour l'analyse des professions - et, en retour, l'intérêt relatif qu'ont manifestés les sociologues des professions pour la théorie bourdieusienne du monde social. Alors que les sociologues américains s'intéressent aux professions depuis les années 1940, il faudra attendre près de trente ans pour voir émerger une « sociologie française » consacrée spécifiquement à cette question. En suivant F. Champy (2009), une des raisons de ce « retard » est à trouver dans l'héritage marxiste qui imprègne la sociologie de l'aprèsguerre en France ; un marxisme dont le contexte intellectuel aura pour effet un « hyperfonctionnalisme » si l'on en croit F. Dubet (2007: 9). La réinscription de cet héritage dans les assises paradigmatiques des développements de la sociologie à cette période en France ${ }^{7}$ permet de saisir le peu d'affinité qu'a la sociologie des professions française avec la théorie de P. Bourdieu, et inversement. Alors que les courants interactionnistes se sont développés aux États-Unis pour partie en opposition à l'approche fonctionnaliste, du côté français, c'est en opposition au marxisme-fonctionnaliste que la « sociologie française des professions » s'est construite. Si l'on ajoute à cela la méfiance qu'a manifesté P. Bourdieu à l'encontre des « taxinomies professionnelles » (Bourdieu, Wacquant, 1992 : 212), l'on saisit le peu d'affinités qu'ont, tant historiquement qu'épistémologiquement, la sociologie de Bourdieu et celle des professions. Plus récemment, c'est une sorte de division des tâches propre au champ sociologique français qui explique en partie le manque de dialogue entre l'analyse dispositionnelle développée par B. Lahire à partir des travaux de P. Bourdieu d'un côté, et la sociologie des professions développée par C. Dubar de l'autre, comme l'explique Muriel Darmon dans l'entretien publié à la fin de ce numéro.

Ceci étant dit, il nous semble venu le temps de dépasser cette opposition et tant les travaux cités ci-dessus que les articles réunis dans ce numéro attestent de la richesse analytique qu'il y a à saisir les professions comme des espaces de positions, à rendre compte des rapports que ces espaces entretiennent avec les univers environnants et à tenir compte des trajectoires sociales des agents qui les composent.

\footnotetext{
${ }^{7}$ Un ancrage qui voit s'opposer une sociologie des professions attachée aux perspectives interactionnistes importées d'outre-Atlantique à une sociologie d'obédience structuraliste largement empreinte d'un héritage marxiste dans le prolongement de laquelle la sociologie de P. Bourdieu s'inscrit.
} 


\subsection{L'analyse dispositionnelle du travail}

Les pratiques de travail sont encadrées par des règles de différentes natures et variablement contraignantes : lois, règlements institutionnels, codes d'éthique et de déontologie, guides de bonnes pratiques. Elles sont façonnées également par les interactions entre professionnels, ainsi qu'avec leurs « clients ». Comme le relèvent C. Avril, M. Cartier et D. Serre (2010), si ces deux éléments (cadres règlementaires et interactions) contribuent à façonner les visions du métier et les pratiques professionnelles, ils agissent néanmoins différemment sur les individus selon leurs positions et trajectoires sociales ou professionnelles. L'appartenance à une institution - au sens le plus large, ici les organisations de travail - est toujours « une forme de "rencontre" dynamique entre ce qui est institué, sous forme de règles, de modalités d'organisation, de savoirs, etc., et les investissements (ou engagements) dans une institution, qui seuls la font exister concrètement » (Lagroye, Offerlé, $2010: 12$ ).

Une étude du travail qui s'appuie sur la théorie des champs et sur une analyse dispositionnelle se doit donc d'accorder une importance prépondérante à la position occupée par les agents au sein de leur espace professionnel, mais également à leur habitus, celui-ci pouvant être modelé, selon des modalités variables, par la socialisation professionnelle. Prendre en compte l'habitus des individus implique de considérer les dispositions activées dans le travail comme pouvant être incorporées dans ou hors des espaces professionnels. Cette manière d'aborder le travail ne remet pas en cause l'étude des cadres règlementaires ou des interactions en situation, mais souhaite les insérer dans la structuration des espaces professionnels et de l'espace social en général. L'analyse dispositionnelle permet en effet d'observer comment des « contextes » de socialisation, à des « moments » spécifiques d'une trajectoire individuelle, produisent des « effets " à d'autres moments (Lahire, 2013 : 117). La manière dont la socialisation professionnelle, en tant que socialisation secondaire, agit sur des individus déjà socialisés, constitue l'objet de ce numéro. Les articles réunis regardent comment des dispositions incorporées antérieurement à l'entrée dans un métier constituent un socle sur lequel agit la socialisation professionnelle et un filtre au travers duquel s'exercent les effets des positions occupées au sein de cet espace. Il s'agit dès lors de nuancer les effets du « passage à travers le miroir » attribué par E. C. Hughes (1958) au processus de socialisation professionnelle, en montrant que celui-ci n'a ni la même forme, ni les mêmes effets sur tous les individus ${ }^{8}$.

\footnotetext{
${ }^{8}$ On pourrait s'intéresser au mécanisme inverse, en regardant comment des dispositions incorporées dans le cadre du travail produisent des effets dans d'autres sphères de vie ou d'autres univers de pratiques, comme l'a fait L. Bernard (2017) à propos des agents immobiliers. Le travail est en effet un « contexte » de socialisation, et sans doute peut-il être considéré comme un contexte de socialisation puissant, tout en étant un espace d'activation de dispositions acquises dans d'autres sphères sociales.
} 
Appréhender les pratiques professionnelles avec le concept d'habitus et non de «culture professionnelle », souvent employé en sociologie des professions, permet de tenir compte des relations entre le travail et les autres sphères de vie, mais également d'éviter un biais dont les usages du concept de culture souffrent généralement : celui que l'on peut qualifier de « mécaniste » (Bourdieu, 1997: 165). La culture est en effet souvent considérée comme une entité extérieure aux individus, s'imposant à eux. Il peut certes arriver que les habitus des agents appartenant à un univers social donné soient similaires, et donc fonctionnent « de manière orchestrée et, si l'on veut, collective » (Bourdieu, 1997 : 186). C'est en ce sens que le concept d'habitus permet également de mettre en lumière de ce qui apparaît comme des «traits culturels » propres à un groupe professionnel, surtout si on l'associe à celui de « doxa professionnelle » (Pichonnaz, 2017) pour rendre compte de ces (éventuelles) dispositions largement partagées.

Comme le relève M. Quijoux, bien que P. Bourdieu se soit très tôt intéressé au travail, la notion d'« habitus professionnel » ne va, quant à elle, pas de soi (Quijoux, 2015 : 51 et suiv.). M. Quijoux insiste à juste titre sur le fait qu'en raison du poids que P. Bourdieu accorde à la socialisation primaire dans le façonnement des trajectoires comme des pratiques, ce que l'on pourrait appeler l'« habitus professionnel » n'est en fait guère plus qu' " une extension et une confirmation de l'habitus de classe » (Quijoux, 2015 : 54). Cependant, à cette lecture d'un habitus des premières heures de la sociologie bourdieusienne qui serait largement congruent avec les positions sociales et professionnelles vers lesquelles il conduit l'agent, on peut préférer une approche processuelle d'un habitus modelé par des expériences successives de socialisations qui peuvent en partie remettre en cause la socialisation primaire. Comme le suggère en effet B. Lahire : « La cohérence des dispositions sociales que peut avoir intériorisées chaque être social dépend [...] de la cohérence des principes de socialisation auxquels il a été soumis » (Lahire, 2012 : 54). En ce qui concerne le concept de « disposition professionnelle », on peut suivre M. Bouvet qui, dans ce numéro, argue qu'une disposition est toujours sociale et jamais strictement professionnelle dans la mesure où, même si elles peuvent effectivement être incorporées dans un contexte professionnel, les dispositions activées dans le travail trouvent fréquemment leur origine en dehors de celui-ci. Ce qui semble spécifique à une profession, toujours selon $\mathrm{M}$. Bouvet, ce sont les savoirs professionnels, que nous proposons d'entendre au sens large : savoirs abstraits ou orientés vers la pratique (savoir-faire), techniques ou attitudes (savoir-être), formalisés ou mobilisés comme compétences. Il est alors pertinent de parler, par exemple, de « savoir-faire professionnel » ou de « compétence professionnelle », pour faire référence à des savoirs qui peuvent être spécifiques à une profession, c'est-à-dire produits et transmis dans un espace professionnel donné?.

\footnotetext{
${ }^{9}$ Les savoirs ne sont cependant pas toujours spécifiques à une profession en particulier : il existe de nombreux chevauchements interprofessionnels. En outre, les savoirs professionnels peuvent reposer, en tous cas en partie, sur des savoirs profanes, en particulier dans les métiers subalternes et les métiers du « care » (Arborio, 2002; Benelli, 2013).
} 
L'analyse dispositionnelle, ou la sociologie de la socialisation, invitent également à regarder les processus mêmes au travers desquels les dispositions sont incorporées par les individus, afin de déterminer quelles instances transmettent quelles dispositions, avec quelle intensité et par quels moyens, comme le fait Émilie Saunier dans ce numéro en explorant la socialisation familiale de l'écrivaine Amélie Nothomb. Ainsi que le relève B. Lahire (2002), la socialisation s'opère au travers de la diffusion et de l'inculcation de normes et de valeurs par des institutions (au sens large : école, travail, famille, médias, etc.), mais également par les pratiques, notamment la participation récurrente à des activités (formalisées ou non), par l'agencement et l'organisation des situations, par le langage oral ou écrit, ou encore par les corps. M. Darmon (2016:100-108) relève également la multiplicité des instances de socialisation : à côté des institutions habituellement prises en compte, il y a bien sûr les pairs, des groupes plus faiblement institutionnalisés, mais également le ou la conjoint·e, des événements politiques ou même «l'effort individuel ». L'auteure évoque non seulement les modalités diverses de socialisation, mais également le fait que celle-ci peut s'exercer de manière variablement consciente et avec une puissance également variable. Ces questions de sociologie de la socialisation restent encore en friche en ce qui concerne l'étude du travail, mais l'on trouvera dans l'entretien avec M. Darmon de nombreuses pistes prometteuses à ce sujet, comme l'étude de la pluralité des socialisations professionnelles, celle de l'accumulation et de la transmission de capitaux au sein des univers professionnels, ou encore celle du rôle socialisateur des sociabilités informelles entre collègues.

\section{Comment le métier (re)façonne les individus. Socialisation primaire et socialisation professionnelle}

Ce numéro se penche particulièrement sur l'impact joué par les socialisations antérieures sur les visions et pratiques du métier. Dans la perspective d'un « emboitement » des socialisations, « ce qui est vécu et intériorisé "avant" devient la base à partir de laquelle est perçu et donc intériorisé ce qui intervient "après" »(Darmon, 2016). Cette assomption essentielle constitue, avec celle de la «transférabilité des dispositions [...] d'un univers à l'autre » (Lahire, $2007: 306$ ), l'hypothèse générale travaillée par tous les auteurs ayant contribué à ce numéro. La socialisation professionnelle étant une socialisation secondaire, elle concerne des individus "dispositionnellement constitués » (cf.l'entretien avec M. Darmon) et s'exerce ainsi sur un «habitus primaire » préexistant. Pour appréhender cette question, les articles réunis ici reposent sur des entretiens approfondis conduits avec des professionnels appartenant à des univers variés : institutions publiques, entreprise privée, champ artistique. Pour rendre compte de limpact des socialisations antérieures sur les visions et pratiques du métier, les méthodes qualitatives, et en particulier les entretiens biographiques, s'imposent en effet (La- 
hire, 2012). Permettant l'analyse fine des trajectoires, ceux-ci donnent accès aux contextes de socialisation qu'ont traversés les individus et permettent d'objectiver l'acquisition ou l'activation de dispositions spécifiques.

Les auteurs explorent à partir de ce matériau les effets de différents contextes de socialisation sur les prises de position professionnelles des agents : socialisation familiale et classe sociale d'origine ; trajectoire sociale, scolaire ou professionnelle antérieure ; socialisation de genre, conjugale, sportive, religieuse ou encore militante. La socialisation familiale, elle-même dépendante de la classe sociale d'origine, est dans ce cadre un contexte de première importance. La plupart des articles montrent que la classe d'origine compte dans la manière dont les individus pratiquent leur métier ou dans leur rapport à celui-ci, de même que leur trajectoire de mobilité sociale, complétant les quelques travaux ayant appréhendé cette question (Avril, 2014 ; Cartier, 2003 ; de Montlibert, 1980 ; Pichonnaz, 2017 ; Serre, 2009 ; Toffel, Longchamp, 2017). Une formation au cirque qui se fait principalement « sur le tas ", éloignée des formes scolaires, attire des individus aux origines sociales modestes, peu dotés en capital scolaire, disposés à se considérer avant tout comme des « artisans », contrairement aux artistes du cirque contemporain, plus souvent issus de familles appartenant à la frange bien dotée en capital culturel des classes supérieures, qui tendent au contraire à valoriser la singularité de leur démarche artistique personnelle (cf. le texte d'É. Salaméro et M. Cordier). Les nouvelles recrues se conformant à l'institution militaire par « résignation » sont aussi celles issues des milieux les plus modestes (cf. le texte de Mélanie Guillaume). La socialisation bourgeoise des «Quants », et le rapport aux mathématiques qui va avec, reste prégnant et contribue à façonner leur rapport au métier et leurs pratiques professionnelles dans la banque (cf. le texte de M. Bouvet). La croyance dans le " pouvoir des mots » et la performativité de la parole de l'écrivaine Amélie Nothomb découle de l'incorporation de dispositions caractéristiques de son milieu social d'origine au travers de différents mécanismes de socialisation familiale (cf. le texte d'É. Saunier).

La question fondamentale découlant de l'analyse des socialisations secondaires est celle du degré de conformité des habitus primaires aux contextes professionnels et, partant, de la nécessité ou de la possibilité, pour pouvoir accomplir le travail, de transformer en partie les habitus primaires - voir même de les convertir. Comme le relèvent M. Darmon dans son entretien et B. Lahire (2013), l'étude des socialisations secondaires doit éviter deux pièges : le premier est de ne pas tenir compte de la socialisation primaire, le second de penser, au contraire, que les socialisations secondaires sont de « simples terrains d'actualisation ou espaces d'épanouissement ou d'expression des dispositions antérieurement acquises » (Lahire, 2013 : 126-127). Les articles réunis ici montrent effectivement que la socialisation professionnelle peut être une socialisation de " renforcement », mais également de «transformation », pour reprendre la distinction introduite par M. Darmon (2016:115-122). Afin d'éviter la nécessité de transformations importantes des habitus, les organisations de travail, entreprises ou institutions pu- 
bliques, peuvent recruter des individus dont l'habitus primaire est déjà ajusté aux exigences de l'organisation et à la doxa professionnelle. Pour ces individus, la socialisation professionnelle est dès lors une socialisation « de renforcement ». C'est le cas pour partie des «Quants»: leurs dispositions de " matheux » ou des dispositions ascétiques, acquises lors de la socialisation familiale au sein des classes supérieures et dans la trajectoire scolaire, peuvent être pleinement activées dans l'univers de la finance (cf. le texte de M. Bouvet). Il paraît cependant difficile d'envisager un processus de recrutement qui puisse infailliblement repérer les dispositions nécessaires à la conformation à l'institution : même l'armée ne parvient pas à recruter uniquement des individus pleinement disposés à « s'en remettre » à elle. Le processus d'intériorisation d'une disposition à se soumettre à l'autorité de l'institution militaire et à s'approprier ses catégories de perception dépend en effet largement de la socialisation antérieure des nouvelles recrues (cf. le texte de M. Guillaume).

Étudier l'articulation entre socialisation primaire et socialisation professionnelle, parallèlement à la continuité des " socialisations de renforcement ", consiste dès lors à observer, d'une part, les phénomènes de transformation des agents, leur permettant de se conformer aux règles et normes dominantes de leur profession, y compris lorsque cela nécessite d'annuler ou de rendre moins actives certaines dispositions. Cela s'observe notamment en ce qui concerne les «Quants » : si la banque épouse bien des aspects de leur socialisation primaire, elle requiert également d'acquérir une disposition « rationnelle-stratégique » se traduisant par exemple par un goût pour la concurrence et la compétition, ce qui ne va pas de soi pour tous les individus recrutés. Comme l'indique B. Lahire, les acteurs peuvent en effet être placés, « simultanément ou successivement, au sein d'une pluralité de mondes sociaux non homogènes, parfois contradictoires" (Lahire, 2012 : 54). Lorsque la profession remet en cause des dispositions incorporées lors de la socialisation primaire, la mise en conformité n'est pas le seul phénomène possible : les professionnels non conformes peuvent remettre en question la doxa professionnelle (Pichonnaz, 2018). Alors que d'anciens militants de la gauche chrétienne investissent un nouvel univers professionnel, ils cherchent ainsi soit à concilier les schèmes de perception incorporés dans leur socialisation militante avec les contraintes de leur nouveau métier, soit défendent plus activement des manières de travailler hétérodoxes (cf. le texte de Gilles Descloux) ${ }^{10}$. L'étude des artistes de cirque montre également comment l'évolution structurelle de ce qu'il convient sans doute de considérer comme une fraction du champ artistique débouche sur une modification des profils de nouvelles et nouveaux entrants. Or, l'accès d'individus ayant des habitus primaires différents, pourvus par exemple de dispositions sportives ou artistiques préexistantes, débouche sur des rapports au travail et des visions du cirque différentes, celles du « cirque contemporain », qui remettent en cause les principes de classement

${ }^{10}$ Notons que l'article de G. Descloux s'intéresse davantage à « l'emboitement » (Darmon, 2016) des socialisations secondaires (religieuse, militante puis professionnelle) qu'à l'impact de la socialisation primaire. 
propres au "nouveau cirque » (cf. le texte d’É. Salaméro et M. Cordier). À ce sujet, comme l'évoque É. Saunier à propos du métier d'écrivaine, certains espaces professionnels peuvent être davantage propices à l'activation de dispositions incorporées dans d'autres univers sociaux, comme le sont, à notre sens, les métiers relationnels s'exerçant sur autrui. L'intérêt que ressentent les individus pour un métier en particulier peut ainsi découler, en tous cas en partie et de manière consciente, de la marge de manœuvre qu'ils estiment pouvoir bénéficier dans un espace professionnel en particulier. C'est l'hypothèse avancée par Gilles Descloux dans sa contribution à propos de la reconversion professionnelle d'anciens militants de la gauche chrétienne.

Par-delà leurs apports avérés, on pourra sans doute regretter que les articles réunis ici n'explorent pas davantage de contextes de socialisation dont on peut imaginer qu'ils contribuent à façonner les visions et pratiques du métier. La socialisation de genre d'abord, dont on sait qu'elle détermine largement les carrières (Battagliola, 1984 ; Lapeyre, Le Feuvre, 2005), mais également les visions du métier et les pratiques professionnelles (Bertaux-Wiame, 2012 ; Paillet, Serre, 2014 ; Pichonnaz, 2017). L'impact des origines ethnoculturelles et des parcours migratoires ensuite, une lacune qui reflète le manque de travaux concernant ce domaine de socialisation dans la sociologie francophone de manière plus générale (Darmon, 2018). Finalement, l'impact de la socialisation politique sur le travail reste également un chantier en cours. La manière dont le travail contribue à façonner le rapport au politique a été étudié, en particulier dans des travaux récents ${ }^{11}$. Il reste néanmoins à rendre compte des influences réciproques entre " visions du monde » et « visions du métier », d'interroger le rôle du rapport au politique, au sens le plus large, dans les choix opérés par les professionnels dans leur travail. Cette question se pose, nous semble-t-il, avec une acuité particulière en ce qui concerne les « métiers relationnels » (Demailly, 2008) ainsi que pour les " professions à pratique prudentielle »(Champy, 2011), puisque leur activité est, comme l'a montré F. Champy (2012), intrinsèquement politique.

Nous avons voulu profiter de ce numéro pour rappeler l'importance de ne pas isoler les professions de l'espace social global, ceci permettant de saisir que les rapports sociaux ne s'arrêtent jamais aux frontières des espaces professionnels. Par ailleurs, il était également l'occasion de (re)dire que l'habitus n'est pas à saisir comme un tout monolithique intransformable, mais comme un patrimoine dispositionnel toujours susceptible de subir les effets des contextes que traversent les agents. Les articles réunis ici, en explorant des contextes de socialisation divers et leurs effets dans des professions variées, attestent du fait que l'analyse de la socialisation antérieure, et plus générale-

\footnotetext{
${ }^{11}$ À ce sujet, voir en particulier : Y. Boughaba (2016), F. Plomb, F. Poglia Mileti (2007), R. Pudal
} (2011), I. Sainsaulieu, M. Surdez (2012), M. Surdez et al. (2016). 
ment l'analyse dispositionnelle, offrent de belles promesses à l'étude du travail en contribuant à renouveler les approches en sociologie des professions. C'est en tous cas notre vœu.

\section{Bibliographie}

Аввотт A. (1988), The system of professions. An essay on the division of expert labor, Chicago/Londres, The University of Chicago Press.

ARboRIo A.-M. (2002), Un personnel invisible. Les aides-soignantes à l'hôpital, Paris, Economica.

AVRIL C. (2014), Les aides à domicile. Un autre monde populaire, Paris, La Dispute.

AvRiL C., Cartier M., Serre D. (2010), Enquêter sur le travail. Concepts, méthodes, récits, Paris, La Découverte.

Battagliola, F. (1984), « Employés et employées. Trajectoires professionnelles et familiales », in M.-A. BARRERE-MAURISSON, Le sexe du travail. Structures familiales et système productif, Saint-Martin-d'Hères, Presses universitaires de Grenoble, p. 5768.

BenelLi N. (2013), « La question des qualifications dans les métiers de care », in M. ModaK, J.-M. Bonvin (dir.), Reconnaître le care : un enjeu pour les pratiques professionnelles, Lausanne, EESP, p. 83-97.

BERCOT R. DivAY S., GADEA C. (dir.) (2012), Les groupes professionnels en tension. Frontières, tournants, régulations, Toulouse, Octares.

BERNARD L. (2017), La précarité en col blanc. Une enquête sur les agents immobiliers, Paris, Presses universitaires de France ( Le lien social »).

BERTAUX-WiAme I. (2012), « Devenir indépendant, une affaire de couple », Cahiers du Genre, vol. 37, p. 13-40.

Boughaba Y. (2016), Citoyennetés populaires en Suisse. Sociabilités et politique à Renens (1945-2013), thèse de doctorat en science politique, Université de Lausanne.

BOURDIEU P. (1984), Questions de sociologie, Paris, Seuil.

BOURDIEU P. (1991), « Le champ littéraire », Actes de la recherche en sciences sociales, vol. 89, p. $3-46$.

BOURDIEU P. (1993), La misère du monde, Paris, Seuil.

BOURDIEU P. (1997), Méditations pascaliennes, Paris, Seuil.

BOURDIEU P. (1998), Les règles de l'art. Genèse et structure du champ littéraire, Paris, Seuil. 
Bourdieu P., Chamboredon J.-C., Passeron J.-C. (1968), Le Métier de sociologue, Paris, Bordas.

BOURDIEU P., WACQUANT J.-D. (1992), Réponses : pour une anthropologie réflexive, Paris, Seuil.

Bucher R., Strauss A. (1961), « Professions in Process », American Journal of Sociology, vol. $66, \mathrm{n}^{\circ} 4$, p. 325-334.

CARTIER M. (2003), Les facteurs et leurs tournées. Un service public au quotidien, Paris, La Découverte.

CHAmpy F. (2009), La sociologie des professions, Paris, Presses Universitaires de France.

Снамpy F. (2011), Nouvelle théorie sociologique des professions, Paris, Presses Universitaires de France.

CHAMPy F. (2012), « Activités professionnelles prudentielles et production de la société », in I. Sainsaulieu, M. Surdez (dir.), Sens politiques du travail. Paris, Armand Colin, p. 57-70.

DARmon M. (2016), La socialisation, Paris, Armand Colin («128»).

DARMON M. (2018), « Socialisation. Petite histoire d'un manuel », Idées économiques et sociales, vol. 191, p. 6-14.

DE Montlibert C. (1980), « L'éducation morale des familles. L'extension du métier de puéricultrice », Actes de la recherche en sciences sociales, vol. 32, n 1, p. 65-76.

Demailly L. (2008), Politiques de la relation. Approche sociologique des métiers et activités professionnelles relationnelles, Villeneuve d'Ascq, Presses Universitaires du Septentrion.

DemazièRE D., Gadea C. (dir.) (2009) Sociologie des groupes professionnels. Acquis récents et nouveaux défis, Paris, La Découverte.

DuBAR C. (2015 [1991]), La Socialisation. Construction des identités sociales et professionnelles, Paris, Armand Colin (« collection U »).

Dubar C., Tripier P., Boussard V. (2011), Sociologie des professions, Paris, Armand Colin (« collection $\mathrm{U} »$ ).

Dubet F. (2007), L'expérience sociologique, Paris, La Découverte.

Hughes E. C. (1958), Men and their work, Glencoe, The Free Press.

Hughes E. C. (1996), Le regard sociologique. Essais choisis, Paris, EHESS.

LAgroye J., Offerle, M. (2010), Sociologie de l’institution, Paris, Belin. 
LAHIRE B. (2002), Portraits sociologiques. Dispositions et variations individuelles, Paris, Armand Colin.

LAHIRE B. (2007), L'esprit sociologique, Paris, La Découverte.

LAHIRE B. (2012 [1995]). Tableaux de familles. Heurs et malheurs scolaires en milieux populaires, Paris, Gallimard/Le Seuil (« Points »).

LAHIRE B. (2013), Dans les plis singuliers du social. Individus, institutions, socialisations, Paris, La Découverte.

LAPEyRe N., Le FeuVRe N. (2005), « Féminisation du corps médical et dynamiques professionnelles dans le champ de la santé », Revue française des affaires sociales, vol. 1, p. 59-81.

LEBARON F. (2000), La croyance économique. Les économistes entre science et politique, Paris, Seuil.

Longchamp P., Toffel K., BÜHlmann F., TAWFIK A. (2018), « L'espace professionnel infirmier : une analyse à partir du cas de la Suisse romande ", Revue française de sociologie, vol. $59, \mathrm{n}^{\circ}$ 2, p. 219-258.

MATHIEU L. (2000), « L’espace de la prostitution. Éléments empiriques et perspectives en sociologie de la déviance », Sociétés contemporaines, $\mathrm{n}^{\circ} 38$, p. 99-116.

Mathieu L. (2007), « L'espace des mouvements sociaux », Politix, vol. 77, p. 131-151.

PAILlET A., SERRE D. (2014), « Les rouages du genre. La différenciation des pratiques de travail chez les juges des enfants », Sociologie du Travail, vol. 56, nº 3, p. 342-364.

Pichonnaz D. (2017), Devenirs policiers. Une socialisation professionnelle en contrastes, Lausanne, Antipodes (« Le livre politique - CRAPUL »).

Pichonnaz D. (2018), « Contester l'ordre professionnel. Les raisons de l'engagement réformateur policier », in F. Bajard, B. Crunel, C. Frau, F. Nicolas, F. Parent (dir.), Professionnalisation(s) et État. Une sociologie politique des groupes professionnels, Villeneuve d'Ascq, Presses Universitaires du Septentrion, p. 211-238.

Pinell P. (2009), « La genèse du champ médical : le cas de la France (1795-1870) », Revue française de sociologie, vol. 2, n 50, p. 315-349.

Pinell P. (2012), « À propos du champ médical : quelques réflexions sur les usages sociologiques du concept de champ ", in F. LEBARON, G. MAUger, Lectures de Bourdieu, Paris, Ellipses, p. 305-318.

Plomb F., Poglia Mileti F. (2007), « Individual expressions of right-wing extremism. Understanding the affinity to radical populism in observing the changes in the work field », in J. FLECKER (dir.), Changing working life and the appeal of the extreme right, Ashgate, Routledge, p. 217-237. 
PUDAL R. (2011), « La politique à la caserne. Approche ethnographique des rapports à la politique en milieu pompier », Revue française de science politique, vol. 61, p. 917-944.

Quijoux M. (dir.) (2015), Bourdieu et le travail, Rennes, Presses universitaires de Rennes.

Sainsaulieu I., Surdez M. (dir.) (2012), Sens politiques du travail, Paris, Armand Colin.

SERRE D. (2009). Les coulisses de l'État social. Enquête sur les signalements d'enfant en danger, Paris, Raisons d'agir.

Surdez M., Zufferey E., Sainsaulieu I., Poglia Mileti F., Plomb F. (2016), L'enracinement professionnel des opinions politiques. Enquête auprès d'agriculteurs, d'ingénieurs et de directeurs de ressources humaines exerçant en Suisse, Zurich/Genève, Seismo.

TOFFEL K., Longchamp P. (2017), « Importer, transformer, diffuser les savoirs infirmiers. Trois figures de la lutte pour l'autonomisation d'une profession », Anthropologie er Santé [En ligne], vol. 14, URL : http://anthropologiesante.revues.org/2536.

TOFFEL K. (2018), Positions et prises de position dans la profession infirmière. La structuration d'un espace professionnel au prisme de la théorie des champs, thèse de doctorat en sciences sociales, Université de Lausanne.

VAUCHEZ A. (2008), « Droit et politique dans la construction européenne », in C. BELOT, P. MAGnette, S. SAURUgGer (dir.), Science politique de l'Union européenne, Economica, p. 53-80.

Vezinat N. (2016), Sociologie des groupes professionnels, Paris, Armand Colin («128»). 
